\title{
Interacción espacial y temporal entre dos mesocarnívoros en un paisaje antropizado del centro de México
}

\section{Spatial and temporal interaction between two mesocarnivores in a human-modified landscape of central Mexico}

\section{Maria Eugenia Espinosa- \\ Flores ${ }^{1}$ (D) \\ Nalleli E. Lara-Díaz ${ }^{2}$ (D) \\ Emma E. Sanchez ${ }^{1}$ [D, \\ Carlos A. López-González ${ }^{1 *}$ (it)}

${ }^{1}$ Laboratorio de Zoología,

Facultad de Ciencias Natu-

rales, Universidad Autónoma de

Querétaro. Av. De las Ciencias

S/N, Col. Juriquilla, CP. 76230 .

Santiago de Querétaro, México.

${ }^{2}$ Soluciones Ambientales Itzen

A.C. Calle Palenque No. 421

int 1, Col. Vértiz Narvarte,

Delegación Benito Juárez. CP.

03600. Ciudad de México, México.

*Autor de correspondencia: cats4mex@gmail.com

Artículo científico

Recibido: 21 de enero de 2019

Aceptado: 30 de agosto de 2019

Como citar: Espinosa-Flores ME, Lara-Díaz NE, Sanchez EE, López-González CA (2020) Interacción espacial y temporal entre dos mesocarnívoros en un paisaje antropizado del centro de México. Ecosistemas y Recursos Agropecuarios 7(1): e2089. DOI: 10.19136/era.a7n1.2089
RESUMEN. Los perros son los carnívoros más abundantes y ampliamente distribuidos en el mundo, con un alto potencial de interactuar y ejercer un efecto negativo sobre la fauna nativa. Se investigó la existencia de segregación espacial y temporal entre perro y gato montés en 40 parches de hábitat del centro de México. Se utilizaron registros fotográficos de 220 estaciones de fototrampeo para analizar sus probabilidades de ocupación, detección y patrones de actividad. La ocupación y detección fueron similares en el área de estudio (perro: $p s i=0.513 \pm$ $0.302, p=0.080 \pm 0.062$, gato montés: $p s i=0.600 \pm 0.473, p=0.052 \pm 0.098$ ), aquellas unidades de muestreo con mayor probabilidad de ocupación de perro tuvieron menor probabilidad de ocupación de gato montés. La ocupación del perro estuvo asociada con la presencia humana y la del gato montés con mayor cobertura arbórea, lejos de caminos y poblados. El gato montés fue primordialmente nocturno, y el perro diurno. Sus patrones de actividad se sobrepusieron un $55 \%$. La mayor sobreposición ocurrió en periodos nocturnos/crepusculares, dónde, se presentó el $38 \%$ de los registros de perro. Este periodo de actividad ocurre generalmente cuando disminuye la actividad humana, por lo que la falta de vigilancia en el área podría incrementar el riesgo de interacciones negativas, como competencia o depredación sobre la fauna nativa. Debido a la fuerte asociación del perro con las actividades humanas, es necesaria una concientización de sus efectos en el ecosistema, como mayor control sobre su presencia, movimientos y reproducción.

Palabras clave: Gato montés, ocupación, patrones de actividad, perro, urbanización.

ABSTRACT. Dogs are the most abundant and widely distributed carnivores worldwide with a high potential to interact and exert a negative effect on native fauna. We investigated the existence of the spatial and temporal segregation between dogs and bobcats in 40 habitat patches across central México. We used photographic records from 220 cameras stations to analyzed their occupancy, detection and activity patterns. Their occupancy and detection were similar across the study area (dog: $p s i=0.513 \pm 0.302, p=0.080 \pm 0.062$, bobcat: $p s i=0.600$ $\pm 0.473, p=0.052 \pm 0.098$ ), within sampling units, those with higher probability of occupancy of dogs had a lower probability of bobcats. Dog's occupancy was associated with human presence while bobcat's with a higher tree cover, and higher distance from roads and human settlements. Bobcats were mainly nocturnal, whereas dogs were diurnal. Their activity patterns overlapped by $55 \%$. The highest overlap occurred within nocturnal/crepuscular periods, where $38 \%$ of dog's records occurred. This nocturnal behavior takes place when human activities decreases, thus, the lack of surveillance in the area could increase the risk of negative interactions such as competition or depredation over the native fauna. Due to the strong association of dogs with human activities, it is necessary to increase the awareness of their effects within the ecosystem, combined with efforts to increase control of the presence, movements and reproduction of dogs.

Key words: Bobcat, occupancy, activity patterns, dog, urbanization. 


\section{INTRODUCCIÓN}

La urbanización es una de las principales causas de perturbación y fragmentación del hábitat (Czech et al. 2000). Actualmente, la expansión urbana es un fenómeno global que amenaza los ecosistemas naturales remanentes y la biodiversidad (McKinney 2008, Seto et al. 2012). Entre los efectos de la urbanización sobre la fauna silvestre se encuentran el desplazamiento inmediato de los individuos fuera de las áreas desarrolladas, incremento del aislamiento entre los individuos que persisten en los parches de hábitat, reducción de la conectividad funcional y diversidad genética, defaunación e incremento de la vulnerabilidad de los parches de hábitat para la colonización de especies invasoras (Hughes y Macdonald 2013, Cushman et al. 2016). Los efectos negativos de las especies invasoras sobre la fauna silvestre nativa se dan principalmente a través de la competencia, depredación y transmisión de enfermedades (Vanak y Gompper 2010, Doherty et al. 2017), y pueden tener efectos en cascada, impactando de manera negativa todo un ecosistema (Courchamp et al. 2003, Ritchie et al. 2013).

El perro (Canis lupus familiaris) es una de las especies invasoras más dañinas para la fauna nativa (Doherty et al. 2016). Debido a su alta asociación con el humano, tanto su presencia como su tamaño poblacional se han incrementado de manera paralela con la de éste (Wandeler et al. 1993, Butler y DuToit 2002), por lo que es el mesocarnívoro más abundante y con mayor distribución en el mundo (Daniels y Bekoff 1989). Tanto los perros ferales, considerados aquellos individuos totalmente independientes, hasta perros cuyos movimientos son dependientes de su dueño (Vanak y Gompper 2010, Ritchie et al. 2013), son capaces de invadir parches de hábitat provocando diferentes efectos en la fauna silvestre. Se han documentado interacciones negativas entre el perro y la fauna nativa, cómo el hostigamiento y la mortalidad de herbívoros silvestres, domésticos (Manor y Saltz 2004, Bergman et al. 2009, Sheriff 2009, Anaya-Zamora et al. 2017), y otras especies de carnívoros como el coyote (Canis latrans), la chilla (Lycalopex griseus), y el gato montés (Lynx rufus)
(Kamler 2003a, Lenth et al. 2008, Silva-Rodríguez et al. 2010). La direccionalidad de la interacción frecuentemente depende del tamaño corporal, siendo la especie de mayor tamaño la dominante, mientras que la de menor tamaño actúa como subordinada (Palomares y Caro 1999, Elbroch y Kusler 2018). Ante la amenaza de mortalidad por parte de una especie dominante, la subordinada tiende a emplear estrategias que le permitan reducir el riesgo de una interacción directa, facilitando la coexistencia entre ambas especies. Estas estrategias frecuentemente implican cambios en el comportamiento de la especie subordinada, lo que resulta en una segregación espacial y/o temporal con la dominante (Harmsen et al. 2009, Elbroch y Kusler 2018, Espinosa-Flores et al. 2018).

En este sentido, se espera que el perro actúe como especie subordinada ante carnívoros de talla grande ( $>20 \mathrm{~kg})$, evitando las áreas donde éstos se encuentren (Morrison et al. 2007). No obstante, pueden llegar a ser competidores y fuente de mortalidad importantes de estas especies (Kamler 2003a, Wierzbowska et al. 2016). Por otra parte, en hábitats dónde los carnívoros de talla grande han sido extirpados, el perro puede fungir como depredador (Ritchie et al. 2013). Además, poseen ventajas sobre los carnívoros silvestres, como percepción positiva por parte de los humanos, tamaños y densidades poblacionales independientes de la abundancia de presas, así como constante reclutamiento de individuos (Kamler 2003b, Vanak y Gompper 2009). Esto convierte al perro en una amenaza, especialmente en ambientes periurbanos, dónde las condiciones bióticas y abióticas por sí mismas podrían no ser las más adecuadas para la persistencia de la fauna nativa a largo plazo, y la presión que podría ejercer el perro sobre sus poblaciones pueden acelerar su extirpación local (Doherty et al. 2017).

El centro de México es una de las áreas que ha perdido la mayor parte de sus ecosistemas naturales por actividades humanas (Challenger 1998, 2014). Una de las áreas con mayores pérdidas es la región del Bajío que comprende alrededor de $50000 \mathrm{~km}^{2}$ de los estados de Querétaro, Guanajuato y Michoacán, en la que viven más de nueve millones de personas 
(Rzedowski y Calderón de Rzedowski 1987, 1991, INEGI 2010a). El área, conformada por grandes valles con pequeñas áreas serranas, es uno de los principales polos de desarrollo del país, donde las actividades agropecuarias, la urbanización e industrialización han reducido los ecosistemas naturales a pequeños parches de vegetación (Villaseñor y Ortiz 2012, Challenger 2014). Este proceso continúa en los estados de Guanajuato y Querétaro, cuyas poblaciones humanas se han incrementado en un $24 \mathrm{y}$ $40 \%$, respectivamente; en los últimos 40 años (INEGI 1970, 2010a), colocándolos entre los 10 estados con mayor densidad poblacional (INEGI 2010a), y como una de las áreas de mayor expansión urbana (Seto et al. 2012).

Actualmente, el proceso de urbanización por el desplazamiento de las áreas de cultivo por áreas industriales, representa una amenaza para la persistencia de la fauna silvestre en los parches de hábitat (Espinosa-Flores y López-González 2017). En este paisaje antropizado, la comunidad de carnívoros nativos está representada por especies generalistas como zorrillos (Conepatus leuconotus, Mephitis macroura, Spilogale angustifrons), prociónidos (Bassariscus astutus, Nasua narica, Procyon lotor), mustélidos (Mustela frenata), félidos (Lynx rufus) y cánidos (Urocyon cinereoargenteus, Canis latrans) (Espinosa-Flores y López-González 2015, EspinosaFlores y López-González 2017). Dentro de esta comunidad, el gato montés representa la especie más susceptible al proceso de la urbanización, por características como bajas densidades, dieta estricta, amplios requerimientos de área y susceptibilidad a la presencia humana (Hansen 2007). Mientras que el perro, al invadir parches de hábitat, puede usar tanto los recursos naturales de los parches como los subsidiados por asentamientos humanos, lo que favorece que sus movimientos no estén restringidos por la expansión urbana (Vanak y Gompper 2010, Morin et al. 2018).

En el área de estudio, se han documentado comportamientos antagonistas entre el perro y el gato montés (López-González et al. 2015), lo que podría desencadenar una evasión espacial y temporal en respuesta a la presencia del perro (George y Crooks 2006, Lenth et al. 2008). Por lo que, la presencia de ambas especies en paisajes urbanizados del centro de México es una oportunidad para determinar el efecto de los perros sobre el gato montés, especie cuya presencia se encuentra en riesgo por la expansión urbana. Por lo que el objetivo del estudio fue evaluar la interacción espacial y temporal entre perros y el gato montés en parches de hábitat en un paisaje antropizado en el centro de México a partir de análisis de ocupación y patrones de actividad de ambas especies.

\section{MATERIALES Y MÉTODOS}

\section{Área de estudio}

El estudio se llevó a cabo en un área aproximada de $10000 \mathrm{~km}^{2}$ de 19 municipios de la porción sur de los estados de Guanajuato y Querétaro (Figura 1). El área presenta un rango de altitud de 1675 a $3350 \mathrm{~m}$, con precipitación y temperatura media anual de $600 \mathrm{~mm}$ y de $17{ }^{\circ} \mathrm{C}$, respectivamente (Vidal-Zepeda 1990a, 1990b). La superficie antropizada con cultivos, asentamientos humanos, suelo desnudo y pastizal inducido constituyen el $72.28 \%$ de la superficie total, los cuerpos de agua el $1.57 \%$, mientras que la vegetación nativa compuesta por bosques, selva baja caducifolia, pastizal halófilo, tular, y matorral crasicaule, representa el $26.15 \%$, de la cual el $76.37 \%$ corresponde a vegetación secundaria, principalmente selva baja caducifolia (INEGI 2017a).

\section{Diseño experimental}

Durante cuatro periodos de muestreo (2014, 2016, 2017 y 2018) se colocaron de cuatro a seis estaciones de fototrampeo en 40 parches de hábitat dentro del área de estudio, con un total de 220 estaciones (Figura 1). La separación espacial entre estaciones de fototrampeo dentro de parches fue entre 500 y $1000 \mathrm{~m}$. Cada estación de fototrampeo consistió de una trampa cámara y un atrayente (mezcla de avena, vainilla y sardina o Carman's Bobcat Gland Lure ${ }^{\mathrm{B}}$ ), el cual consideramos que no afecta el estado de ocupación del sitio (du Preez et al. 2014, Shannon et al. 2014, Gomes-Rocha et al. 2016). Las 


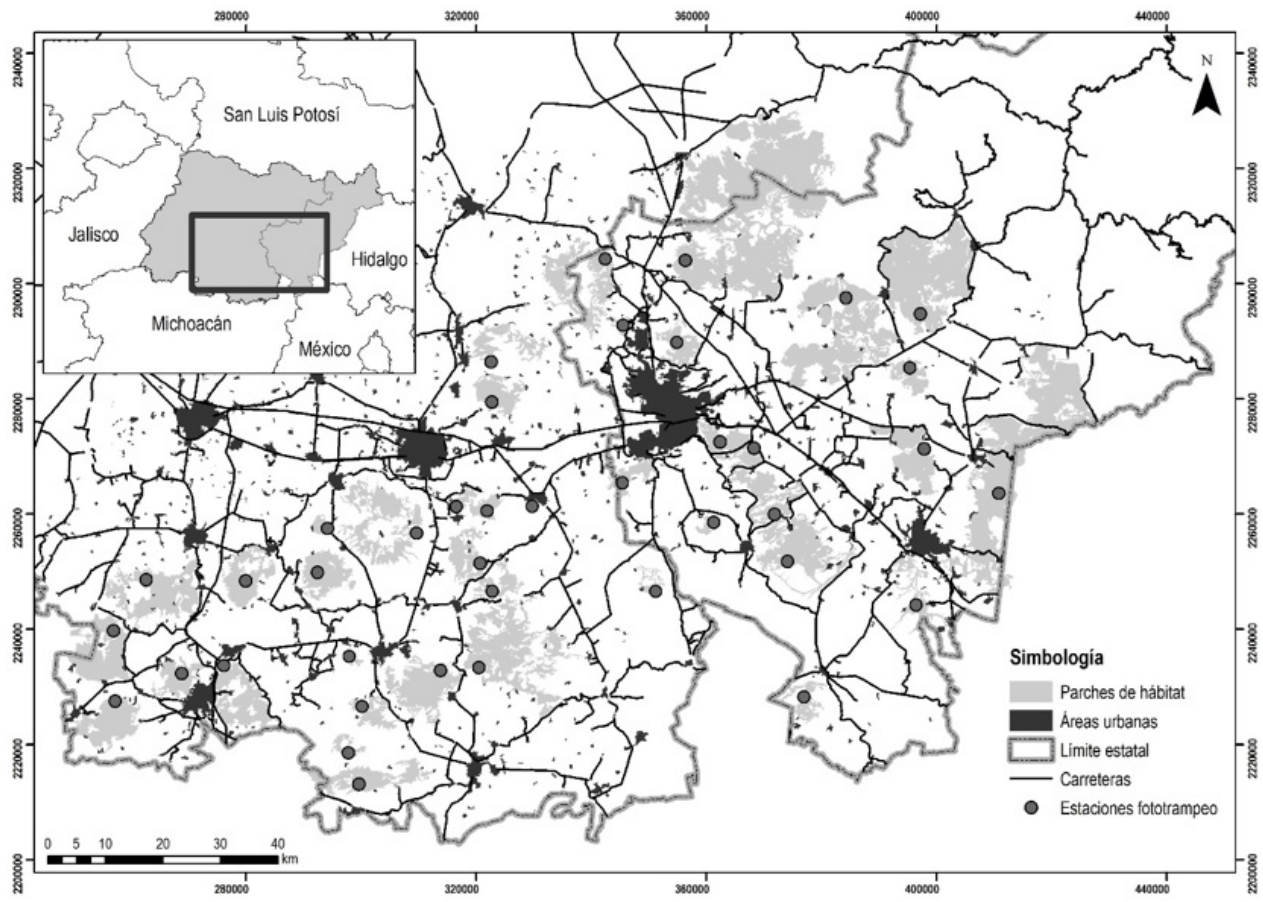

Figura 1. Localización del área de estudio, incluyendo los parches de hábitat muestreados, áreas urbanas y una estación de fototrampeo que representa el grupo de trampas cámara usadas por cada parche de hábitat.

trampas cámara se programaron para activarse cada minuto si había movimiento o calor, y capturar tres eventos fotográficos sucesivos, tuvieron un periodo de actividad de siete a 20 días; variación que dependió del riesgo de robo del equipo. El esfuerzo total de muestreo fue de 3315 días cámara.

\section{Modelos de ocupación \\ Probabilidad de ocupación y detección}

La probabilidad de ocupación (psi) y detección (p) para el perro y el gato montés se determinó de manera independiente, a partir de la información de registros fotográficos obtenidos en las 220 estaciones de fototrampeo (Farris et al. 2015) durante un periodo promedio de actividad de $15.06 \pm 3.27$ días. Se desarrolló un historial de detección para cada especie considerando 20 ocasiones de muestreo (equivalentes a 20 días de monitoreo); en general el periodo de actividad de cada cámara en las 20 ocasiones de muestreo fue variable. Las unidades de muestreo correspondieron a cada estación de fototrampeo $(n=$ 220). En el historial se asignó 0 cuando la especie objetivo no fue detectada durante un día/cámara, 1 cuando la especie objetivo fue detectada, y NA cuando en la unidad y periodo de muestreo no hubo una trampa cámara activa (MacKenzie et al. 2006).

Para cada especie se construyeron 62 modelos divididos en dos grupos: 31 modelos considerando sólo covariables para la probabilidad de ocupación y 31 modelos con covariables únicamente para la probabilidad de detección. Las covariables $(n=5)$ se incluyeron de acuerdo con todas las combinaciones (interacciones) posibles. Adicionalmente, se desarrolló un modelo nulo (sin covariables). Los modelos se implementaron en el programa $R$ ver. 3.2.2, a través de la función occu del paquete unmarked (Fiske et al. 2015), utilizando el modelo de ocupación para una especie, y para una sesión de monitoreo (Mackenzie et al. 2002, 2006). Una vez generados los modelos, se seleccionaron los tres mejor ajustados de cada grupo (ocupación/detección), con base en el criterio de información de Akaike (AIC; Akaike 1973, Burnham y Anderson 2002). Posteriormente, se construyeron 
nueve modelos a partir de la combinación de los modelos seleccionados previamente, considerando juntas las probabilidades de ocupación y detección. A partir de todos los modelos generados $(n=72)$ se identificaron los modelos mejor ajustados, los cuales se promediaron de acuerdo en el peso del AIC (wAIC, Burnham y Anderson 2002). Con base en el promedio de los estimadores beta del modelo se calculó la $p$ si y $p$ para la especie objetivo en cada unidad de muestreo; los valores de probabilidad de ocupación y detección en el área de estudio se estimaron promediando los valores $p s i$ y $p$ de cada unidad de muestreo.

Para determinar diferencias significativas en la probabilidad de ocupación de las especies en las mismas unidades de muestreo, se dividieron en cinco grupos con base en las probabilidades de ocupación del perro; los grupos tuvieron intervalos de psi de $0.2(0 \leq 0.2,0.2 \leq 0.4,0.4 \leq 0.6,0.6 \leq 0.8,0.8 \leq$ 1). A cada psi del perro se le asoció la psi para el gato montés que se presenta en la misma unidad de muestreo, y por grupo se les aplicó una prueba de $t$ pareada con el programa PAST ver. 3.01 (Hammer et al. 2001).

\section{Covariables}

Se consideraron diferentes covariables que podrían tener efecto en las probabilidades de ocupación y detección de gato montés y perros, las cuales se utilizaron para construir los modelos de ocupación. Estas covariables fueron: 1) distancia a poblados (INEGI 2010b) considerada como la distancia topográfica de cada estación de fototrampeo al poblado más cercano (Ordeñana et al. 2010); la distancia topográfica toma en consideración la altitud entre sitios, por lo que constituye una mejor percepción de la accesibilidad humana a los puntos de muestreo, en contraste con una distancia lineal. 2) Distancia a caminos pavimentados (INEGI 2017b), los cuales son evitados por el gato montés, mientras que pueden facilitar el acceso a los parches de hábitat por parte de los perros (Doherty et al. 2015, Reed et al. 2017). 3) Cobertura arbórea (Sexton et al. 2013), considerada una característica de hábitat importante para la presencia de gato mon- tés (Kolowski y Wolf 2002, Espinosa-Flores y LópezGonzález 2017). Estas tres variables se calcularon con el programa ArcMap 10.1 (ESRI 2013). 4) índice de éxito de captura de humanos, y 5) índice de éxito de captura de ganado vacuno; ambos índices se calcularon como eventos independientes ( $24 \mathrm{~h}$ ) entre el número de días de actividad de cada estación de fototrampeo por 100 (Farris et al. 2014). La selección de la variable de éxito de captura de los seres humanos, obedece a la alta asociación que presenta con el perro y se ha documentado que las veredas usadas por los humanos reducen su uso por el gato montés (Wandeler et al. 1993, George y Crooks 2006, Lenth et al. 2008); el uso del ganado vacuno se debe a que en el área de estudio suele pastar de modo extensivo y tiende a pasar mucho tiempo frente a la cámara, lo que potencialmente podría afectar la probabilidad de detección del perro y gato montés.

\section{Patrones de actividad}

Para obtener una medida de sobreposición en los periodos de actividad entre ambas especies, se consideraron como eventos independientes a las fotografías separadas por un periodo mínimo de una hora (Lara-Díaz et al. 2018). Posteriormente, para poder comparar entre las horas a lo largo de diferentes años y sitios de estudio, estas fueron convertidas a hora solar con el paquete solaR (Perpiñán 2012, Foster et al. 2013). El paquete overlap se usó para calcular un coeficiente de sobreposición $(\Delta 1$, con base en el número de observaciones; Meredith y Ridout 2014), el cual tiene un rango de valores de cero (sin sobreposición) a uno (sobreposición total), con sus intervalos de confianza (IC) al 95\% a partir de 10000 muestras bootstrap. Todos los análisis se llevaron a cabo en el programa $R$ ver. 3.2.2.

\section{RESULTADOS}

Para el perro, se obtuvieron 1089 fotografías (160 eventos independientes) en 79 de las 220 estaciones de fototrampeo colocadas (35.90\%). En total se detectó a la especie en 34 de los 40 parches de hábitat (85\%). Para el gato montés, se obtuvieron 229 fotografías (44 eventos independientes) en 36 
estaciones de fototrampeo (16.36\%) a lo largo de 24 parches de hábitat $(60 \%)$. El perro y el gato montés se detectaron de forma conjunta en 21 parches de hábitat $(52.5 \%)$, pero sólo se detectó su coocurrencia en 18 estaciones de fototrampeo de las 96 colocadas en esos parches. El intervalo de tiempo entre detecciones de ambas especies en la misma estación tuvo un rango de cinco a $258 \mathrm{~h}$. En cuatro ocasiones, ambas especies se detectaron el mismo día, con periodo de diferencia de cinco a $15 \mathrm{~h}$.

\section{Ocupación}

Los modelos que mejor explicaron la probabilidad de ocupación y detección para el perro ( $n=8$ modelos con wAIC acumulado igual a 1) incluyeron las covariables para psi de distancia a caminos, éxito de captura de humanos, y éxito de captura de vacas en todos los modelos, distancia a poblados ( $\mathrm{n}=5$ modelos) y cobertura arbórea $(\mathrm{n}=2$ modelos). Para la $p$, las covariables presentes en todos los modelos fueron éxito de captura de humanos, éxito de captura de vacas y distancia a poblados; la cobertura arbórea y la distancia a caminos se encontraron explicando menos modelos ( $n=6$ y 3 modelos, respectivamente). De acuerdo al promedio de los estimadores beta de estos modelos, el éxito de captura de humanos es la covariable de mayor influencia que aumenta la probabilidad de ocupación del perro; el resto de las covariables tienen una influencia reducida en comparación con el éxito de captura de humanos. Solo una menor distancia a poblados y mayor cobertura arbórea disminuyen la probabilidad de ocupación del perro. En la probabilidad de detección también la covariable de éxito de captura de humanos fue la de mayor influencia aumentando la probabilidad de detección, aunque con magnitud comparable con el resto de las covariables; la mayor distancia a caminos y a poblados disminuye la probabilidad de detección (Tabla 1).

Para el gato montés, la mayoría de los modelos construidos tuvieron que ser promediados con relación al peso del AIC ( $n=66$ modelos con wAIC acumulado igual a 1). Ninguna de las covariables estuvo presente en todos los modelos: para $p s i$, distancia a caminos ( $\mathrm{n}=19$ modelos), éxito de captura de humanos $(n=14)$, éxito de captura de vacas $(n=20)$, distancia a poblados $(n=19)$, cobertura arbórea $(\mathrm{n}=18)$; para $p$ distancia a caminos ( $\mathrm{n}$ $=16)$, éxito de captura de humanos $(n=13)$, éxito de captura de vacas $(n=22)$, distancia a poblados $(n=12)$, cobertura arbórea $(n=16)$. La cobertura arbórea fue la covariable con mayor influencia sobre las probabilidades de ocupación y detección (Tabla 1).

Para toda el área de estudio, las probabilidades de ocupación y detección entre ambas especies fueron similares. Para el perro se obtuvo una $p s i=0.513 \pm 0.302$ y $p=0.080 \pm 0.062$, mientras que para el gato montés una psi $=0.600 \pm 0.473$, y $p=0.052 \pm 0.098$. Al dividir las probabilidades de ocupación en grupos con intervalos de probabilidad de 0.2, se encontraron diferencias significativas en la mayoría de los grupos (Tabla 2), de modo que existe una tendencia a que las unidades de muestreo que presentan una mayor probabilidad de ocupación de perro presenten una menor probabilidad de ocupación de gato montés (Figura 2).

\section{Patrones de actividad}

Se obtuvieron 221 eventos independientes de perro y 46 de gato montés. El perro exhibió una actividad principalmente diurna, pero el $38 \%$ de su actividad ( $n=84$ registros) se presentó durante los periodos nocturnos y crepusculares. Mientras que el gato montés mostró un patrón de actividad nocturno (20:00-05:00 h), y crepuscular (06:00-07:00 y 18:0019:00 h), mientras que el pico de su actividad diurna (08:00-17:00 h) se dio principalmente en el periodo de las 12:00-13:30 h. El coeficiente de sobreposición de sus patrones de actividad fue de $\Delta 1=0.55, \mathrm{IC}=$ 0.40-0.65; el mayor pico en su sobreposición ocurre entre las 04:00-06:00 h (Figura 3).

\section{DISCUSIÓN}

Los resultados sugieren la existencia de una segregación espacial y temporal entre el perro y gato montés en diferentes grados dentro del área de estudio. La segregación espacial, sólo fue observada a nivel de las unidades de muestreo, donde aquellas con mayor probabilidad de ocupación para el perro 
Tabla 1. Promedio de los valores beta para la estimación de probabilidades de ocupación y detección del perro $(n=8$ modelos promediados) y gato montés ( $\mathrm{n}=66$ modelos promediados).

\begin{tabular}{lcccc}
\hline \multirow{2}{*}{ Covariable } & \multicolumn{2}{c}{ Perro } & \multicolumn{2}{c}{ Gato montés } \\
& $p s i$ & $p$ & $p s i$ & $p$ \\
\hline Intercepto & 6.65 & -2.59 & 7.92 & -3.79 \\
Distancia a caminos & 0.69 & -0.10 & 9.28 & 0.55 \\
Éxito de captura de humanos & 19.92 & 0.34 & 4.39 & 0.05 \\
Éxito de captura de ganado vacuno & 0.28 & 0.29 & 0.05 & 0.19 \\
Distancia a poblados & -0.19 & -0.21 & 3.39 & 0.05 \\
Cobertura arbórea & -0.36 & 0.27 & 19.93 & 1.31 \\
\hline
\end{tabular}

Tabla 2. Prueba de t pareada para evaluar diferencias significativas en la probabilidad de ocupación entre el perro y gato montés a partir de las mismas unidades de muestreo. Los grupos se dividieron con base en la probabilidad de ocupación del perro (intervalos de 0.2).

\begin{tabular}{ccccc}
\hline Intervalo de probabilidad & $\mathrm{n}$ (pares) & \% de unidades de muestreo & $\mathrm{t}$ & $\mathrm{p}$ \\
\hline $0 \leq 0.2$ & 22 & 10.00 & -7.52 & $<0.0001$ \\
$0.2 \leq 0.4$ & 87 & 39.55 & -4.96 & $<0.0001$ \\
$0.4 \leq 0.6$ & 46 & 20.91 & -2.00 & 0.05 \\
$0.6 \leq 0.8$ & 10 & 4.55 & 1.65 & 0.132 \\
$0.8 \leq 1$ & 55 & 25.00 & 5.90 & $<0.0001$ \\
\hline
\end{tabular}

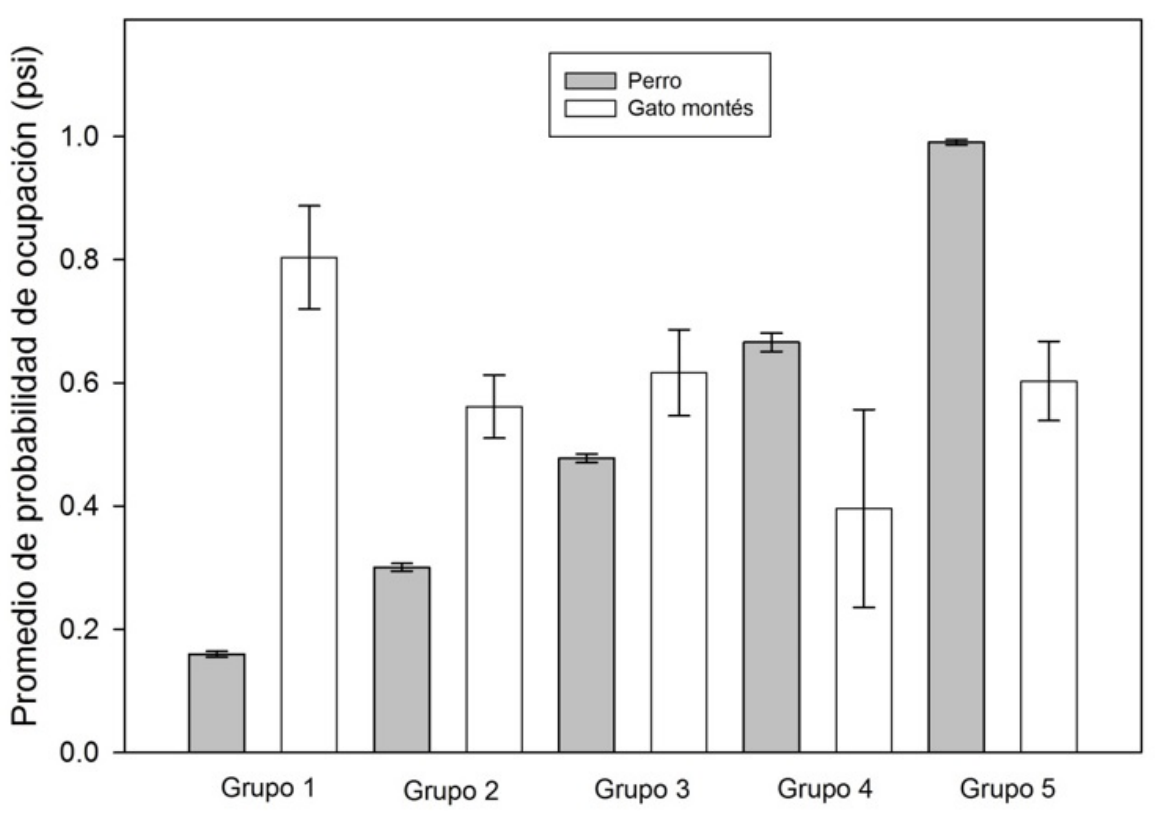

Figura 2. Segregación espacial de perro y gato montés (Promedio \pm error estándar de psi) en las mismas unidades de muestreo. La definición de grupos de unidades de muestreo $(n=5)$ es con base en intervalos de 0.2 de probabilidades de ocupación para el perro.

presentaron una menor probabilidad de ocupación del gato montés (Figura 2). Esta escala puede indicar condiciones de microhábitat como cambios en cobertura arbórea, disponibilidad de agua y disponibilidad de presas (Kolowski y Woolf 2002); que son capaces de determinar la distribución y actividades de los or- ganismos (Putman y Wratten 1984). Sin que representen las condiciones generales a nivel de paisaje, donde perro y gato montés no presentan diferencias en su probabilidad de ocupación.

Las diferencias entre la probabilidad de ocupación en las mismas unidades de muestreo (Tabla 2) 


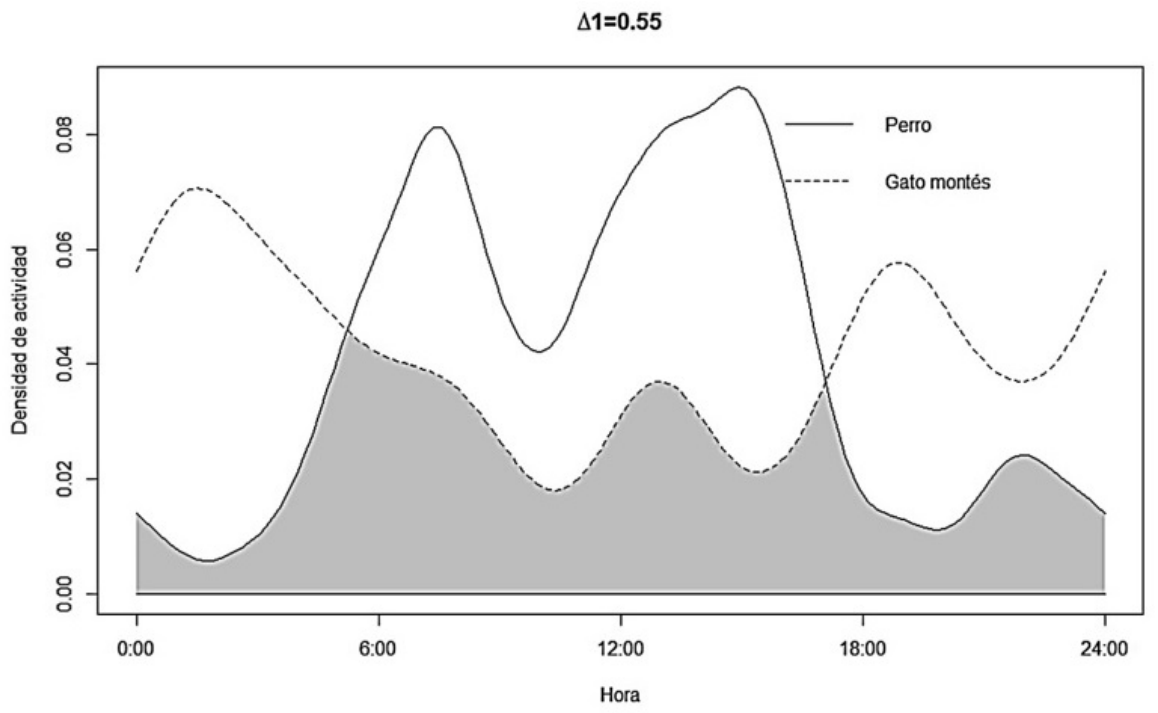

Figura 3. Coeficiente de sobreposición de los patrones de actividad entre perro y gato montés. La sobreposición está representada por el área gris.

pueden estar asociadas a que el gato montés percibe al perro como una amenaza potencial (George y Crooks 2006, Lenth et al. 2008). En otros ambientes antropizados, se ha reportado que el perro puede ser una causa de mortalidad importante del gato montés (Lembeck 1986, Knick 1990) y, debido a que sus ataques frecuentemente están dirigidos hacia individuos juveniles y hembras, implica un posible impacto a nivel poblacional, ya que las hembras son la fuente de nuevos individuos (Donovan et al. 2011).

El perro presentó mayor probabilidad de ocupación y detección en sitios más perturbados. La cobertura arbórea disminuye su probabilidad de ocupación, lo que es consistente con el uso y posible selección de áreas abiertas respecto a sitios cerrados (Sepúlveda et al. 2015, Morin et al. 2018). Por lo que, el cambio de uso de suelo para dar paso a coberturas antropizadas podría facilitar sus movimientos de colonización e invasión (Vanak y Gompper 2010). La distancia a poblados, no tuvo influencia en su ocupación, a pesar de que se ha documentado como una de las variables más importantes para predecir la presencia del perro en los parches de hábitat (Vanak y Gompper 2010). El éxito de captura de los humanos fue la variable de mayor influencia, lo que refleja la fuerte asociación que tienen con las actividades antropogénicas en el área de estu- dio (Sepúlveda et al. 2015), y la importancia de los humanos para actuar como facilitadores o inhibidores de las interacciones del perro con la fauna silvestre (dos Santos et al. 2018, Home et al. 2018).

En contraste, las probabilidades de ocupación y detección del gato montés fueron mayor en los sitios menos perturbados (Tabla 1). La cobertura arbórea representó la variable con mayor influencia, lo que refleja su afinidad hacia áreas de mayor calidad, y susceptibilidad a la pérdida de hábitat (Kelly et al. 2016, Espinosa-Flores y López-González 2017). La distancia a caminos fue la segunda variable con mayor influencia, donde una mayor distancia aumenta la probabilidad de ocupación. El bajo flujo de vehículos en los caminos del área de estudio no es una barrera para el movimiento del gato montés, pero los caminos pueden representar mayor accesibilidad para el perro y los humanos, generando perturbaciones (Kerley et al. 2002, Sepúlveda et al. 2015).

El perro presentó una actividad primordialmente diurna, lo que refleja su asociación con los humanos y sus actividades (Farris et al. 2015, Wang et al. 2015). La actividad del perro disminuyó durante los periodos nocturnos y crepusculares (Figura 3), cuando también disminuye la actividad humana. La actividad diurna del perro puede ser un riesgo para carnívoros y sus presas que se encuentran activos en 
el mismo periodo dentro del área de estudio, cómo el caso del gato montés, coyote (Espinosa-Flores et al. 2018), y conejo castellano (Sylvilagus floridanus, Chapman et al. 1980).

El gato montés, a pesar de que puede estar activo en cualquier hora del día (Anderson y Lovallo 2003), presentó mayor actividad en la noche, probablemente por la presencia del perro y de las actividades humanas que ocurren en el área de estudio como cacería, colecta de leña, pastoreo, recreación y tala (George y Crooks 2006, Tigas et al. 2002, Wang et al. 2015). Exhibir un comportamiento primordialmente nocturno, podría asociarse con ajuste de sus patrones de actividad a causa de presiones antropogénicas, lo que ha sido señalado como una de las respuestas de la fauna silvestre ante la expansión de la huella humana (Gaynor et al. 2018). Esta modificación en los patrones de actividad constituye una posible disminución en su adecuación, ya que se ha documentado que su éxito de cacería es bajo en noches con poca iluminación lunar; ante esto el gato montés generalmente incrementa sus movimientos diurnos para obtener alimento (Rockhill et al. 2013). Sin embargo, en un escenario donde el perro se encuentra activo durante el día, la búsqueda y obtención de alimento del gato montés durante este periodo podría verse limitada.

Se espera que las especies de carnívoros nativas e invasoras exhiban patrones de actividad opuestos como resultado de interacciones negativas como competencia y depredación (Vanak y Gompper 2010, Elbroch y Kusler 2018). Pero, no existe un consenso para determinar el rango de valores que constituye una baja o alta sobreposición temporal. Al respecto, Farris et al. (2015) encontraron un rango de sobreposición de $\Delta_{1}=0.23-0.88$ entre perros y otros carnívoros nativos de Madagascar, por lo que sugieren que una alta sobreposición podría indicar un incremento en las interacciones y competencia entre perros y especies nativas. En el presente estudio, se observó una tendencia de disminución en la actividad del gato montés, al incrementar la del perro (Figura 3). Por la sobreposición de patrones de actividad media entre perro y gato montés, y la segregación espacial alta, por la diferencia en probabilidades de ocupación en las mismas unidades de muestreo, se puede inferir que más que una mayor proporción de interacciones, puede existir un desplazamiento del gato montés provocado por el perro. En el caso de sobreposición en sus patrones de actividad, durante los periodos nocturnos y crepusculares, los perros activos durante estas horas podrían no estar asociados con los humanos, y por lo tanto ejercer mayor efecto sobre la fauna silvestre, incluyendo al gato montés (Weston y Stankowich 2014, Young et al. 2018). Al ser una especie común en este tipo de paisajes antropizados, puede ejercer un efecto negativo en la composición de las comunidades, así como la distribución y abundancia de la fauna nativa (Vanak y Gompper 2010, Lessa et al. 2016, Farris et al. 2017, Morin et al. 2018). Esto aunado a las ventajas que tiene el perro, cómo son abundancias independientes de las presas, reclutamiento constante de individuos (Kamler 2003b, Vanak y Gompper 2009), formación de grupos que favorecen la competencia y depredación (Butler et al. 2004, Wierzbowska et al. 2016). Una percepción positiva por parte del humano y tolerancia hacia su comportamiento depredador, los convierten en amenazas para la fauna silvestre, más en ambientes fragmentados con incremento continuo de urbanización.

\section{CONCLUSIONES}

La probabilidad de segregación espacial y temporal producida por el perro hacia otros mesocarnívoros como el gato montés, con efectos en la fauna silvestre ha sido previamente documentado. En este estudio, el gato montés y el perro tuvieron una segregación espacial y temporal, y una respuesta diferencial a las perturbaciones antropogénicas. Debido a la alta asociación del perro con las actividades humanas, es fundamental concientizar a los dueños de los perros y/o a los habitantes locales que coexisten con la fauna silvestre, sobre los impactos que el perro puede ocasionar, ya que muchas de las interacciones entre fauna silvestre y perros ocurren cuando no se encuentran acompañados por humanos. Se recomienda implementar medidas cómo la esterilización de los perros para evitar 
el reclutamiento continúo de individuos y restringir su acceso a los parches de hábitat; en caso extremo se debe considerar la aplicación de la eutanasia a los individuos con mayor potencial de impacto, así como a aquellos que se han vuelto ferales. Además, se deben incrementar los estudios sobre el comportamiento y ecología del perro a escala de paisaje e incluir sus efectos dentro de la dinámica del ecosistema, a nivel de poblaciones y comunidades. Conociendo aspectos básicos como su dieta, patrones de movimiento y densidades, se puede tener un panorama más amplio sobre los efectos de esta especie invasora sobre la fauna silvestre.

\section{AGRADECIMIENTOS}

Este estudio fue financiado por The Summerlee Foundation de 2016-2018 y al Consejo Nacional de Ciencia y Tecnología (Convocatoria SEP-Ciencia Básica 2017, No: 287607) durante el 2018. A los habitantes de las distintas localidades y ejidos por el permiso de trabajar en sus propiedades y por su asistencia durante los muestreos. A la Universidad Autónoma de Querétaro y a las siguientes personas por su asistencia en el trabajo de campo: V. Agoitia, A. Arroyo, K. Camargo, F. Cruz, F. Espinosa, M. Gómez, C. Guzmán, A. Leal, F. Mora, A. Ocaña, S. Paz, C. Rojas, K. Saucedo, M. Sosa, S. Tafoya, A. Torres, D. Zamora. A R. Jones por revisar el manuscrito; y al CONACyT por proporcionar una beca a MEEF (No: 464614) y EES (No: 469089).

\section{LITERATURA CITADA}

Akaike $H$ (1973) Information theory and an extension of the maximum likelihood principle. En: Petrov BN, Csaki $\mathrm{F}(\mathrm{ed})$. Proceedings of the 2 nd International Symposium on Information Theory. Akademiai Kiado. Budapest, Hungría. pp: 267-281.

Anaya-Zamora V, López-González CA, Pineda-López RF (2017) Factores asociados en el conflicto humanocarnívoro en un área natural protegida del centro de México. Ecosistemas y Recursos Agropecuarios 4: 381-393.

Anderson EM, Lovallo MJ (2003) Bobcat and Lynx. En: Feldhamer GA,Thompson BC, Chapman JA (ed). Wild mammals of North America: biology, management, and conservation. 2th edition. Johns Hopkins University Press. Baltimore, USA. pp: 758-786.

Bergman DL, Breck SW, Bender SC (2009) Dogs gone wild: feral dog damage in the United States. Proceedings of the 13th Wildlife Damage Management Conference 13: 177-183.

Burnham KP, Anderson DR (2002) Model selection and multimodel inference: a practical information-theoretic approach. Springer Science \& Business Media. New York, USA. 485p.

Butler JRA, Du-Toit JT (2002) Diet of free-ranging domestic dogs (Canis familiaris) in rural Zimbabwe: implications for wild scavengers on the periphery of wildlife reserves. Animal Conservation 5: 29-37.

Butler JRA, Du-Toit JT, Bingham J (2004) Free-ranging dogs (Canis familiaris) as predators and prey in rural Zimbawe: threats of competition and disease to large wild carnivores. Biological Conservation 115: 369378.

Challenger A (1998) Utilización y conservación de los ecosistemas terrestres de México: pasado, presente y futuro. Comisión Nacional para el Uso y Conocimiento de la Biodiversidad, Instituto de Biología de la UNAM y Agrupación Sierra Madre S.C. Ciudad de México, México. 847p. 
Challenger A (2014) Paisajes naturales y humanos de México. En: Valdez R, Ortega-S JA (ed). Ecología y manejo de fauna silvestre en México. Colegio de Postgraduados. Estado de México, México. pp: 21-52.

Chapman JA, Hockman JG, Ojeda MM (1980) Sylvilagus floridanus. Mammalian species 136: 1-8.

Courchamp F, Chapuis JL, Pascal M (2003) Mammal invaders on islands: Impact, control and control impact. Biological reviews of the Cambridge Philosophical Society 78: 347-383.

Cushman SA, McRae BH, McGarigal K (2016) Basics of landscape ecology: an introduction to landscape and population processes for landscape genetics. En: Balkenhol N, Cushman SA, Storfer AT, Waits LP (ed). Landscape genetics: concepts, methods, applications. John Willey \& Sons. West Sussex, UK. pp: 11-34.

Czech B, Krausman PR, Devers PK (2000) Economic associations among causes of species endangerment in the United States. BioScience 50: 593-601.

Daniels TJ, Bekoff M (1989) Population and social biology of free-ranging dogs, Canis familiaris. Journal of Mammalogy 70: 754-762.

Doherty TS, Dickman CR, Nimmo DG, Ritchie EG (2015) Multiple threats, or multiplying the threats? Interactions between invasive predators and other ecological disturbances. Biological. Conservation 190: 60-68.

Doherty TS, Glen AS, Nimmo DG, Ritchie EG, Dickman CR (2016) Invasive predators and global biodiversity loss. Proceedings of the National Academy of Sciences of the United States of America 113: 11261-11265.

Doherty TS, Dickman C.R, Glen AS, Newsome TM, Nimmo DG, Ritchie EG, Vanak AT, Wirsing J (2017) The global impacts of domestic dogs on threatened vertebrates. Biological Conservation 210: 56-59.

Donovan TM, Freeman M, Abouelezz H, Royar K, Howard A, Mickey R (2011) Quantifying home range habitat requirements for bobcats (Lynx rufus) in Vermont, USA. Biological Conservation 144: 2799-2809.

dos Santos CL, LePendu Y, Giné GA, Dickman CR, Newsome TM, Cassano CR (2018) Human behaviors determine the direct and indirect impacts of free-ranging dogs on wildlife. Journal of Mammalogy 99: 1261-1269.

du Preez BD, Loveridge AJ, Macdonald DW (2014) To bait or not to bait: a comparison of camera-trapping methods for estimating leopard Panthera pardus density. Biological Conservation 176: 153-161.

Elbroch LM, Kusler A (2018) Are pumas subordinate carnivores, and does it matter? PeerJ 6: e4293.

Espinosa-Flores ME, Lara-Díaz NE, López-González CA (2018) Respuesta a un paisaje antropizado por el género Canis en el centro de México. En: Ramírez-Bautista A, Pineda-López R (ed). Ecología y Conservación de Fauna en Ambientes Antropizados. REFAMA-CONACyT-UAQ. Querétaro. México. pp: 5-15.

Espinosa-Flores ME, López-González CA (2015) Range extension for the White-nosed coati (Nasua narica) in Southeastern, Guanajuato, México. Western North American Naturalist 75: 244-247.

Espinosa-Flores ME, López-González CA (2017) Landscape attributes determine bobcat (Lynx rufus escuinapae) presence in Central México. Mammalia 81: 101-105.

ESRI (2013) ArcGIS Desktop: Release 10.1. Environmental Systems Research Institute. Redlands, California.

Farris ZJ, Gerber BD, Karpanty S, Murphy A, Andrianjakarivelo V, Ratelolahy F, Kelly MJ (2015) When carnivores roam: temporal patterns and overlap among Madagascar's native and exotic carnivores. Journal of Zoology 296: 45-57.

Farris ZJ, Karpanty SM, Ratelolahy F, Kelly MJ (2014) Predator-primate distribution, activity, and co-occurrence in relation to habitat and human activity across fragmented and contiguous forests in Northeastern Madagascar. International Journal of Primatology 35: 859-880. 
Farris ZJ, Kelly MJ, Karpanty S, Murphy A, Ratelolahy F, Andrianjakarivelo V, Holmes C (2017) The times they are a changin': Multi-year surveys reveal exotics replace native carnivores at a Madagascar rainforest site. Biological Conservation 206: 320-328.

Fiske I, Chandler R, Miller D, Royle A, Kery M, Hostetler J, Hutchinson R, Smith A, Kellner K (2015) Package 'unmarked': models for data from unmarked animals. https://cran.r-project.org/web/packages/unmarked/. Fecha de consulta: 7 de junio de 2018.

Foster VC, Sarmento P, Solman R, Torres N, Jácomo AT, Negroes N, Fonseca C, Silveira L (2013) Jaguar and puma activity patterns and predator-prey interactions in four Brazilian biomes. Biotropica 45: 373-379.

Gaynor KM, Hojnowski CE, Carter NH, Brashares JS (2018) The influence of human disturbance on wildlife nocturnality. Science 360: 1232-1235.

George SL, Crooks KR (2006) Recreation and large mammal activity in an urban nature reserve. Biological Conservation 33: 107-117.

Gomes-Rocha D, Ramalho EE, Magnusson WE (2016) Baiting for carnivores might negatively affect capture rates of prey species in camera-trap studies. Journal of Zoology 300: 205-2012.

Hammer $\varnothing$, Harper DAT, Ryan PD (2001) PAST: Paleontological Statistics Software Package for Education and Data Analysis. Paleontologia Electronica 4(1) Art. 9.

Hansen K (2007) Bobcat, master of survival. Oxford University Press. New York, USA. 212p.

Harmsen BJ, Foster RJ, Silver SC, Ostro LET, Doncaster CP (2009) Spatial and temporal interactions of sympatric jaguars (Panthera onca) and pumas (Puma concolor) in a Neotropical forest. Journal of Mammalogy 90 : 612-620.

Home C, Bhatnagar YV, Vanak AT (2018) Canine Conundrum: domestic dogs as an invasive species and their impacts on wildlife in India. Animal Conservation 21: 275-282.

Hughes J, Macdonald DW (2013) A review of the interactions between free-roaming domestic dogs and wildlife. Biological Conservation 157: 341-352.

INEGI (1970) IX Censo general de población. Instituto Nacional de Estadística y Geografía. http://www.beta.inegi. org.mx/proyectos/ccpv/1970/. Fecha de consulta: 8 de junio de 2018.

INEGI (2010a) Censo de población y vivienda 2010. Instituto Nacional de Estadística y Geografía. http://www.beta.inegi.org.mx/proyectos/ccpv/2010/default.html. Fecha de consulta: 8 de junio de 2018.

INEGI (2010b) Localidades de la República mexicana. 2010. Instituto Nacional de Estadística y Geografía. http://www.conabio.gob.mx/informacion/gis/. Fecha de consulta: 8 de junio de 2018.

INEGI (2017a) Carta de uso de suelo y vegetación, Serie VI. Instituto Nacional de Estadística y Geografía. http://www.inegi.org.mx/geo/contenidos/recnat/usosuelo/. Fecha de consulta: 8 de junio de 2018.

INEGI (2017b) Conjunto de datos vectoriales de carreteras y vialidades urbanas edición 1.0. Instituto Nacional de Estadística y Geografía. http://www.inegi.org.mx/geo/contenidos/topografia/vectoriales_carreteras.aspx. Fecha de consulta: 8 de junio de 2018.

Kamler JF, Ballard W, Gipson PS (2003a) Occurrence of feral dogs (Canis lupus familiaris) in northwest Texas: an observation. Texas Journal of Agriculture and Natural Resources 16: 75-77.

Kamler JF, Keeler K, Wiens G, Richardson C, Gipson PS (2003b) Feral dogs, Canis familiaris, kill Coyote, Canis latrans. Canadian Field Naturalist 117: 123-124. 
Kelly M, Morin D, López-González CA (2016) Lynx rufus. The IUCN Red List of Threatened Species http://www.iucnredlist.org/details/12521/0. Fecha de consulta: 10 de junio de 2018.

Kerley LL, Goodrich JM, Miquelle DG, Smirnov EN, Quigley HB, Hornocker MG (2002) Effects of roads and human disturbance on Amur tigers. Conservation Biology 16: 97-108.

Knick ST (1990) Ecology of bobcats relative to exploitation and a prey decline in southeastern Idaho. Wildlife Monographs 108: 1-42.

Kolowski JM, Woolf A (2002) Microhabitat use by bobcats in southern Illinois. Journal Wildlife Management 66 : 822-832.

Lara-Díaz NE, Coronel-Arellano H, López-González CA, Sánchez-Rojas G, Martínez-Gómez JE (2018) Activity and resource selection of a threatened carnivore: the case of black bears in northwestern Mexico. Ecosphere 9: e01923. Doi: 10.1002/ecs2.1923.

Lembeck M (1986) Long term behavior and population dynamics of an unharvested bobcat population in San Diego County. En: Miller SD, Everett DD (ed). Cats of the world: biology, conservation, and management. National Wildlife Federation. Washington D.C., USA. pp: 305-310

Lenth BE, Knight RL, Brennan ME (2008) The effects of dogs on wildlife communities. Natural Areas Journal 28 : 218-227.

Lessa I, Guimarães TCS, de Godoy Bergallo H, Cunha A, Vieira EM (2016) Domestic dogs in protected areas: a threat to Brazilian mammals? Natureza \& Conservação 14: 46-56.

López-González CA, Ávila-Aguilar D, Cruz-Torres MF (2015) Abundancia del gato montés (Lynx rufus escuinapae J.A. Allen, 1903) en el Parque Nacional El Cimatario, Querétaro, México. Acta Zoológica Mexicana 31: 138140.

MacKenzie I, Nichols JD, Lachman GB, Droege S, Royle JA, Langtimm CA (2002) Estimating site occupancy rates when detection probabilities are less than one. Ecology 83: 2248-2255.

MacKenzie DI, Nichols JD, Royle JA, Pollock KH, Bailey LA, Hines JE (2006) Occupancy estimation and modeling. Academic Press. Burlington, USA. 344p.

Manor R, Saltz D (2004) The impact of free-roaming dogs on gazelle kid/female ratio in a fragmented area. Biological Conservation 119: 231-236.

McKinney ML (2008) Effects of urbanization on species richness: a review of plants and animals. Urban Ecosystems 11: 161-176.

Meredith M, Ridout M (2014) Package 'overlap': Estimates of coefficient of overlapping for animal activity patterns. https://cran.r-project.org/web/packages/overlap/index.html. Fecha de consulta: 7 de junio de 2018.

Morin DJ, Lesmeister DB, Nielsen CK, Schauber EM (2018) The truth about cats and dogs: Landscape composition and human occupation mediate the distribution and potential impact of non-native carnivores. Global Ecology and Conservation 15: e00413. Doi: 10.1016/j.gecco.2018.e00413.

Morrison JC, Sechrest W, Dinerstein E, Wilcove DS, Lamoreux JF (2007) Persistence of large mammal faunas as indicators of global human impacts. Journal of Mammalogy 88: 1363-1380.

Ordeñana MA, Crooks KR, Boydston EE, Fisher RN, Lyren LM, Siudyla S, et al. (2010) The effects of urbanization on carnivore species distribution and richness. Journal of Mammalogy 91: 1322-1331.

Palomares F, Caro TM (1999) Interspecific killing among mammalian carnivores. The American Naturalist 153: 492-508. 
Perpiñán LO (2012) solaR: solar radiation and photovoltaic systems with R. Journal of Statistical Software 50: 1-32.

Putman RJ, Wratten SD (1984) Principles of ecology. University of California Press. California, USA. 388p.

Ritchie EG, Dickman CR, Letnic M, Vanak AT (2013) Dogs as predators and trophic regulators. En: Gompper ME (ed). Free ranging dogs and wildlife conservation. Oxford University Press. New York, USA. pp: 55-68.

Reed GC, Litvaitis JA, Callahan C, Carroll RP, Litvaitis MK, Broman DJA (2017) Modeling landscape connectivity for bobcats using expert-opinion and empirically derived models: how well do they work? Animal Conservation 20: 308-320.

Rockhill AP, DePerno CS, Powell RA (2013) The effect of illumination and time of day on movements of bobcats (Lynx rufus). PLoS One 8: e69213. Doi: 10.1371/journal.pone.0069213.

Rzedowski J, Calderón de Rzedowski G (1987) El bosque tropical caducifolio de la región mexicana del Bajío. Trace 12: 12-21.

Rzedowski J, Calderón de Rzedowski G (1991) Presentación y guía para los autores y normas editoriales. Flora del Bajío y de Regiones Adyacentes I: 1-14.

Sepúlveda M, Pelican K, Cross P, Eguren A, Singer R (2015) Fine-scale movements of rural free-ranging dogs in conservation areas in the temperate rainforest of the coastal range of southern Chile. Mammalian Biology 80: 290-297.

Seto KC, Güneralp B, Hutyra LR (2012) Global forecasts of urban expansion to 2030 and direct impacts on biodiversity and carbon pools. Proceedings of the National Academy of Sciences 109: 16083-16088.

Sexton JO, Song XP, Feng M, Noojipady P, Anand A, Huang C, et al. (2013) Global, 30-m resolution continuous fields of tree cover: Landsat-based rescaling of MODIS Vegetation Continuous Fields with lidar-based estimates of error. International Journal of Digital Earth 6: 427-448.

Shannon G, Lewis JS, Gerber BD (2014) Recommended survey designs for occupancy modelling using motionactivated cameras: insights from empirical wildlife data. PeerJ 2: e532. Doi: 10.7717/peerj.532.

Sheriff MJ, Krebs CJ, Boonstra R (2009) The sensitive hare: sublethal effects of predator stress on reproduction in snowshoe hares. Journal of Animal Ecology 78: 1249-1258.

Silva-Rodríguez EA, Ortega-Solís GR, Jiménez JE (2010) Conservation and ecological implications of the use of space by chilla foxes and free-ranging dogs in a human-dominated landscape in southern Chile. Austral Ecology 35: 765-777.

Tigas LA, Van Vuren DH, Sauvajot RM (2002) Behavioral responses of bobcats and coyotes to habitat fragmentation and corridors in an urban environment. Biological Conservation 108: 299-306.

Vanak AT, Gompper ME (2009) Dogs Canis familiaris as carnivores: their role and function in intraguild competition. Mammal Review 39: 265-283.

Vanak AT, Gompper ME (2010) Interference competition at the landscape level: the effect of free-ranging dogs on a native mesocarnivore. Journal of Applied Ecology 47: 1225-1232.

Vidal-Zepeda R (1990a) Precipitación media anual, escala 1:4000000. En: Precipitación. Tomo II, Sección IV, 4.6. Atlas Nacional de México (1990-1992). Instituto de Geografía, UNAM. México.

Vidal-Zepeda R (1990b) Temperatura media, escala 1:4000000. En: Temperatura media. Tomo II, Sección IV, 4.4. Atlas Nacional de México (1990-1992). Instituto de Geografía, UNAM. México. 
Villaseñor JL, Ortiz E (2012) La familia Asteraceae en la flora del Bajío y de regiones adyacentes. Acta Botánica Mexicana 100: 259-291.

Wandeler Al, Matter HC, Budde A, Kappeler A (1993) The ecology of dogs and canine rabies: a selective review. Revue Scientifique et Technique Office International des Epizooties 12: 51-71.

Wang Y, Allen ML, Wilmers CC (2015) Mesopredator spatial and temporal responses to large predators and human development in the Santa Cruz Mountains of California. Biological Conservation 190: 23-33.

Weston MA, Stankowich T (2014) Dogs as agents of disturbance. En: Gompper ME (ed). Free-ranging dogs and wildlife conservation. Oxford University Press. Oxford, UK. pp: 94-116.

Wierzbowska IA, Hędrzak M, Popczyk B, Okarma H, Crooks KR (2016) Predation of wildlife by free-ranging domestic dogs in Polish hunting grounds and potential competition with the grey wolf. Biological Conservation 201: 1-9.

Young JK, Bergman DL, Ono M (2018) Bad dog: feral and free-roaming dogs as agents of conflict. Animal Conservation 21: 285-286. 